excretion of a strongly alkaline urine, which may persist for a period of twenty-four hours or more. The alkalinity is undoubtedly due to the presence of carbonates since addition of acid to the urine causes effervescence of carbon dioxide. After the urine has resumed its usual acid character a subsequent injection of morphine may fail to elicit an alkaline urine although the hydrogen ion concentration may be diminished appreciably. On the day of morphine introduction there is usually a definite increase in the elimination of the total urinary nitrogen.

Rabbits fasted until they excrete a strongly acid urine show no significant change in the hydrogen ion concentration of the urine nor is the total nitrogen altered even though very large doses (8o mg. per kilo) of morphine are subcutaneously introduced. These results are in accord with the well-known resistance of rabbits to morphine narcosis.

\title{
54 (I232)
}

\section{The determination of oxygen in blood.}

\section{By Donald D. Van Slyke.}

[Hospital of the Rockefeller Institute for Medical Research, N. Y.]

This requires 5 minutes for human blood, unless a little sapanin has been added to the ammonia, in which case 15 seconds may suffice for the laking.

The apparatus for determining carbon dioxid in blood, described in the Proceedings for the meeting April 21, 1915, can be used with equal facility and accuracy for determination of oxygen. 6 c.c. of ammonia made by diluting the concentrated solution with 200 parts of water, are introduced into the apparatus with 5 drops of caprylic alcohol. The apparatus is evacuated and the air extracted from the ammonia by shaking for a few seconds. The extracted air is expelled and the process completed to make certain that none is left.

2 c.c. of blood are then introduced. The blood and ammonia are mixed and allowed to stand until the blood is laked. Then half a c.c. of saturated potassium ferricyanide solution is in- 
troduced (the cyanide solution is made air-free by boiling or by shaking in an evacuated flask, and is kept in a burrette under a layer of paraffin oil two or three centimeters thick to exclude air). The apparatus is now evacuated, shaken and the oxygen set free determined exactly as is carbon dioxid. The solubility of oxygen in water is so slight that no correction is made for what remains in solution. The only correction necessary is for the small amount of nitrogen gas which 2 c.c. of blood contain. 